\title{
Long-term sequelae after acquired pediatric hemorrhagic cerebellar lesions
}

\author{
Kevin Wingeier • Sandra Bigi • Marwan El-Koussy • \\ Theda Heinks-Maldonado • Eugen Boltshauser • \\ Maja Steinlin
}

Received: 23 August 2010 / Accepted: 30 November 2010 /Published online: 29 December 2010

(C) Springer-Verlag 2010

\begin{abstract}
Purpose The aim of the present study was to assess cognitive, affective, and motor long-term sequelae after acquired focal pediatric cerebellar lesions.

Methods Eight patients with a history of isolated acquired hemorrhagic cerebellar lesions before the age of 13 participated in this study. All participants underwent a neurologic examination, including the Zurich Neuromotor Assessment (ZNA) and the International Cooperative Ataxia Rating Scale (ICARS). Cognitive functions have been evaluated with a general cognitive assessment and an extensive neuropsychological battery. Furthermore, patients and parents filled in questionnaires about quality of life and possible behavioral or emotional problems.

Results The results revealed that all patients exhibited motor problems (ZNA). Most participants had further restricted oculomotor movements (ICARS). Age at injury and the full scale IQ were significantly positively correlated (Pearson
\end{abstract}

K. Wingeier $(\bowtie) \cdot$ S. Bigi $\cdot$ T. Heinks-Maldonado $\cdot$ M. Steinlin

Department of Pediatric Neurology (J212),

University Children's Hospital, Inselspital,

3010 Bern, Switzerland

e-mail: kevin.wingeier@gmail.com

URL: www.insel.ch

K. Wingeier

Division of Experimental Psychology and Neuropsychology,

University of Bern,

Bern, Switzerland

M. El-Koussy

Diagnostic and Interventional Neuroradiology,

University Hospital, Inselspital,

Bern, Switzerland

E. Boltshauser

Pediatric Neurology, University Children's Hospital,

Zurich, Switzerland correlation $0.779 ; p=0.023)$. Conversely, no overall neuropsychological profile could be identified except for marginally reduced reaction times and susceptibility to interference. In addition, borderline results in semantic and phonemic word fluency tasks were apparent. A dysexecutive syndrome was diagnosed in one patient. However, verbal performance and reading abilities were non-pathologic in all participants. The patients reported having a good quality of life without major physical restrictions.

Conclusions Emotional disturbances and the presence of a mild cerebellar cognitive affective syndrome (as frequently described in adult patients) could only be confirmed in adolescents with vermis lesions. Nevertheless, in laboratory conditions, neuropsychological impairments were present in all patients. Heterogeneity of age at injury and exact lesion site may have led to interpersonal differences in neuropsychological outcome.

Keywords Cerebellum · Neuropsychology · Child .

Cognition $\cdot$ Quality of life $\cdot$ Hemorrhage

\section{Introduction}

Since the middle of the last century, the cerebellum has been found to be associated with the coordination and timing of actions, the control and modulation of movements, and the adjustment of posture [1]. At the end of the 1980 s, reports suggesting a possible contribution of the cerebellum to higher-level cognition [2, 3]. In the last two decades, an increasing number of studies have described cognitive impairments following damage to the cerebellum. Some reports have linked cerebellar lesions to difficulties in various cognitive domains, e.g., language and speech problems [4-8], visuo-spatial impairments [8-10], execu- 
tive dysfunctions and (verbal) working memory problems [11-13], learning disabilities $[8,10,12]$, as well as impaired sequencing in cognitive domains $[14,15]$. In 1998, Schmahmann and Sherman tried to combine some of the described symptoms under the term cerebellar cognitive affective syndrome [16]. This syndrome represents a neurobehavioral phenomenon that includes various neuropsychological deficits combined with behavioral/affective symptoms [16]. Symptom-lesion mapping, neuroanatomical and functional neuroimaging studies provide further indications that the cerebellum is involved in cognitive functioning [17-24]. Some studies have even found connections between lesions of the posterior lateral cerebellar hemispheres and higher order cognitive problems, whereas injuries involving the vermis of the cerebellum are accompanied by dysregulation of affect [25-28].

However, the extent of influence of the cerebellum on developing cognitive functions is still insufficiently investigated. Despite the fact that neurocognitive problems after acquired pediatric cerebellar lesions have been described, reports about the emergence of cerebellar cognitive affective syndrome in this population are scarce [28-31]. Nevertheless, many authors stress the importance of an intact cerebellum for the development of neuropsychological functions [24, 32-34]. Thus far, it is known that children who suffer from congenital vermal aplasia (Joubert-Boltshauser syndrome) have severe cognitive deficits [35]. Other reports have linked an underdevelopment of the cerebellum in preterm babies with potential cognitive problems later in life [36-38]. The majority of pediatric studies focusing on cognition after acquired cerebellar lesions have mainly recruited patients suffering from oncological diseases. Neoplastic pediatric cerebellar lesions have repeatedly been found to be associated with different neuropsychological problems [24, 28, 29, 32, 39].

To the best of our knowledge, no systematic evaluation of long-term sequelae after acquired non-neoplastic cerebellar lesions during development has yet been reported. Therefore, in the present study, we focus primarily on the long-term outcomes for neuropsychological functions, quality of life, and possible behavioral/emotional problems in a circumscribed patient group with a history of acquired non-neoplastic cerebellar lesion during childhood.

\section{Methods}

After approval by the Cantonal Ethics Committees of Bern and Zurich, Switzerland, databases of the University Children's Hospitals in Bern and Zurich and the Swiss Neuropaediatric Stroke Registry were systematically searched for former patients with a history of acquired isolated ischemic or hemorrhagic cerebellar lesion under the age of 16 in the past 10 years [40]. Patients younger than 6 years at the time of examination were excluded due to the limited number of neuropsychological tests available for this age group and missing normative data of healthy children. Patients with psychiatric disorders prior to injury, with neurological or systemic disorders potentially interfering with developing cognitive functions and patients with oncological problems were excluded as well. Finally, eight patients qualified for participation. These patients had all suffered from a hemorrhagic cerebellar stroke due to an arterial venous malformation (AVM) or a cerebellar cavernoma (see Table 1). They were thoroughly informed about the aim of the study. Written informed consent was obtained from all participants and their parents.

\section{Neuroimaging}

All participating families granted the research team full access to the children's medical documents, including CT, MRI scans, or angiography images. All initial and followup neuroimaging data were reviewed. The cerebellum was examined in regard to the affected vascular territory, the exact lesion site in the cerebellar hemisphere, and potential lesions in the vermis.

Neurological and neuropsychological assessment

The neurological and neuropsychological assessments were done at least 12 months post-injury. The neurological examination involved a complete Zurich Neuromotor Assessment battery (ZNA) and the International Cooperative Ataxia Rating Scale (ICARS) [41, 42]. Individual neuropsychological assessment was conducted in multiple sessions according to a standardized format and in the same order for all participants. To gain the patient's neuropsychological profiles, the following test materials were chosen: Wechsler Intelligence Scale for Children, 4th (WISC-IV) Edition, or Wechsler Intelligence Scale for Adults, 3rd Edition (WAIS-III), Rey Osterrieth Complex Figure (Rey Figure), Regensburger Word Fluency Test (RWT), a clinical screening of reading abilities (as no reading test covered the whole age range in our patient group - different reading test have been used), Wisconsin Card Sorting Test (WCST), Rey Auditory Verbal Learning Test (RAVLT), Rey Visual Design Learning Test (RVDLT), Corsi Block Tapping Test (CORSI), 5-point Test, DelisKaplan Executive Function System (D-KEFS), and the subtests for alertness, selective attention, divided attention, incompatibility, and working memory from the of the Test Battery of Attentional Performance (TAP). All of these tests are frequently used in clinical trials and in scientific research - are age-scaled - and the test manuals provide normative data of healthy children. 


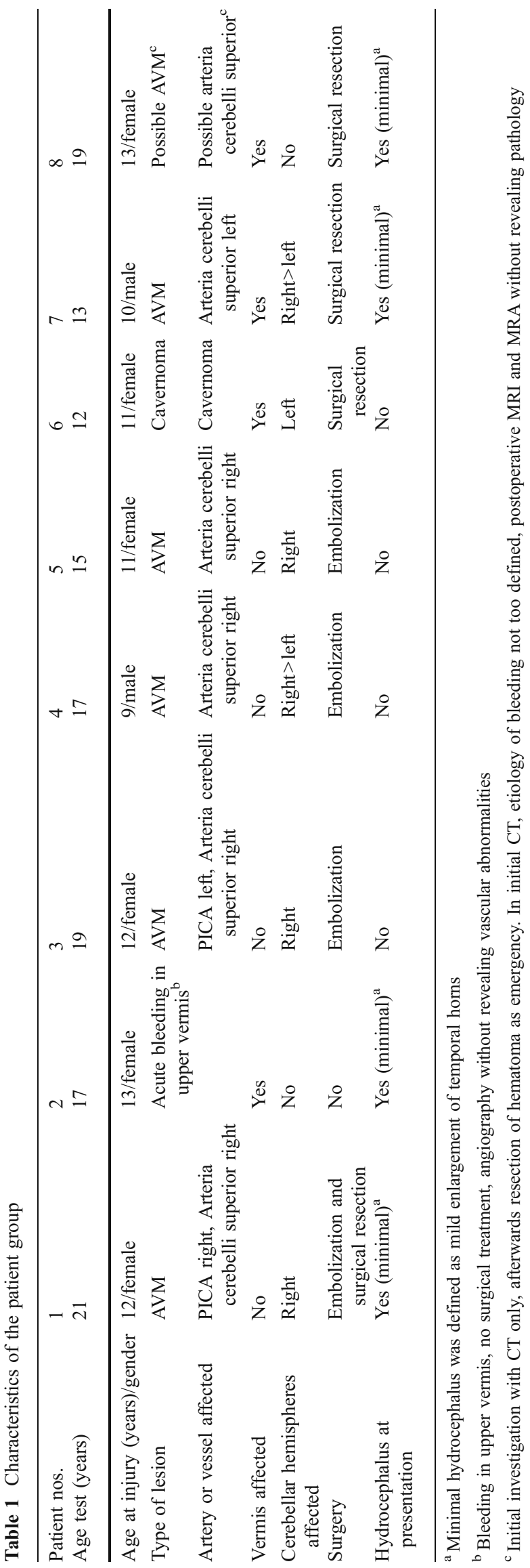

Questionnaires

The patients and their parents were further asked to fill in questionnaires about the patient's physical well-being, emotional health, and possible behavioral problems, as well as the patient's present quality of life. To address these issues, the following questionnaires have been chosen: Child Behaviour Check List (CBCL), Youth Self Report (YSR), the Marburger inventory to establish the quality of life in children and adolescents (ILK-MARSYS), and the Kidscreen-52 long-version questionnaire.

\section{Results}

The patients' characteristics, as well as the etiology of the cerebellar injury, are described in Table 1. The eight patients who were willing to participate were between the ages of 11 and 21 (mean age 16 years and 6 months; SD 3 years and 2 months). The age at the time of manifestation of the cerebellar injury was between 9 and 13 years of age, with a mean age of 11 years. In five participants, the right cerebellar hemisphere has been injured; in two of those patients, the left side was mildly affected, too. A single patient had an isolated lesion on the left cerebellar hemisphere. In $50 \%$ of the patient group, the vermis of the cerebellum was affected. Two of those patients had an isolated vermis injury with no involvement of the cerebellar hemispheres. Minimal hydrocephalus (defined as mild enlargement of temporal horns) was present in four patients at acute presentation. Figures 1 and 2, which show MR images of two selected patients, provide a clear picture of the exact lesion sites and indicate the medical interventions needed in these cases.

Neurological and neuropsychological assessment

Detailed results of the neurological assessments are shown in Table 2. The results revealed the presence of mainly unspecific motor problems (ZNA) and slight symptoms of ataxia (ICARS). Every single patient had results in at least one subtest of the ZNA that were more than one standard deviation below the age-scaled norm. Seven patients even had abnormal results in three or more subtests of the ZNA. The subscales for adaptive fine motor tasks (mean -3.4 ; SD 3.3) and for adaptive gross motor tasks (mean -2.7 ; SD 1.6) were most sensitive. In contrast, motor speed - assessed with the D-KEFS - was average or even above average in all patients (mean 12; SD 1). The ICARS revealed only minor and quite heterogeneous signs of ataxia. Most abnormal results were obtained in the subscale oculomotor movements.

General intelligence assessments of the patient group resulted in a mean full scale IQ (FSIQ) of 103 (SD 11, range 83-118). Only one participant had an FSIQ below 85. Mean 
a)

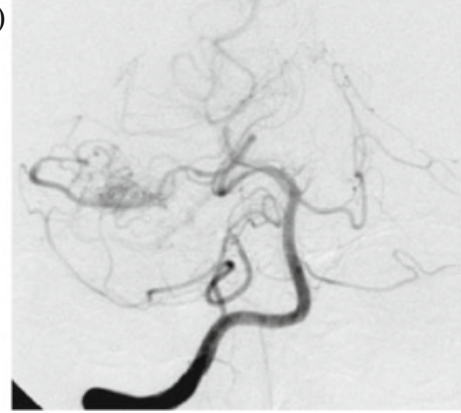

b)

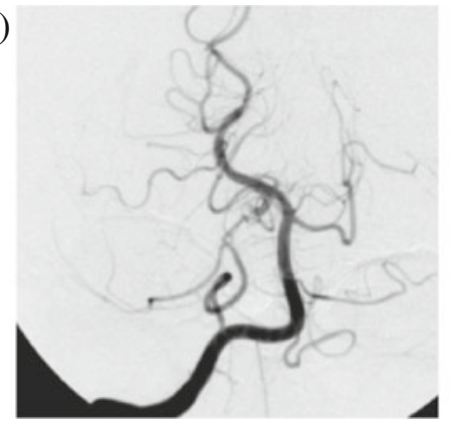

c)

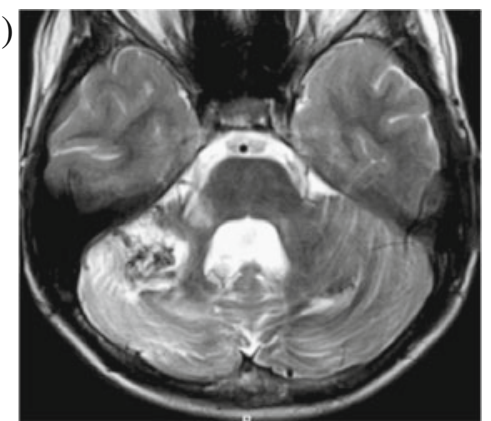

Fig. 1 MR images: Arteriovenous malformation (AVM) in the posterior fossa of patient nos. 4. Digital subtraction angiography, right vertebral artery injection. a Main feeder was the right superior cerebellar artery. b Successful selective embolization. The AVM is no longer opacified. In the c T2-weighted MRI image, notice the parenchymal loss and dark hemosidering deposits in the right and to a lesser extent in the left cerebellar hemispheres as well as in the right middle cerebellar peduncle verbal IQ (VIQ) measured by WAIS-III was 106, whereas the mean performance IQ (PIQ) was 103, with no significant difference between VIQ and PIQ. Age at injury and the FSIQ were significantly positively correlated (Pearson correlation $0.779 ; p=0.023$ ). Thus, young age at the time of injury is associated with lower general cognitive functioning. No significant correlations were found between time since injury (in years) and FSIQ (Pearson correlation 0.027; $p=0.950$ ). A selected overview of the neuropsychological results is illustrated in Table 3. Prolonged reaction time was revealed in the TAP subtests for alertness and for selective attention, where up to $50 \%$ of study sample had pathological results. In the TAP subtest for incompatibility (designed to measure a person's susceptibility to interference), $50 \%$ of the participants showed reaction times of more than one standard deviation below the mean of the norm population (percentile $<16)$. Clinically, no patient showed problems reading aloud (judged by accuracy and speed). Grammar and spelling were not impaired. No confabulations, perseverations, or dysarthric speech could be identified. However, four patients had pathological results in either the semantic or the phonemic verbal fluency task.

On the individual level, some results are worth highlighting in detail. Patient nos. 4 suffering from a superior arterial cerebellar malformation with lesions on the right and left cerebellar hemispheres and a diagnosis of a dysexecutive syndrome had severe deficits in the WAISIII subtest on picture arrangement (more than two standard deviations below the norm), recalling the Rey Figure, all verbal fluency tasks and the Wisconsin Card Sorting Test (scoring below the 9th percentile on all outcome measures). Patient nos. 6, who had a lesion on the left cerebellar hemisphere after a cavernoma, showed insecurities in several memory tasks (e.g., recall of the Rey Figure, CORSI, and RAVLT). Similar problems were discovered in patient nos. 7, who had lesions on both cerebellar hemispheres (right side lesion was bigger than the one on the left). This patient had severe (working) memory problems, e.g., TAP subtest for working memory, RAVLT, RVDLT, and recalling the Rey Figure. a)

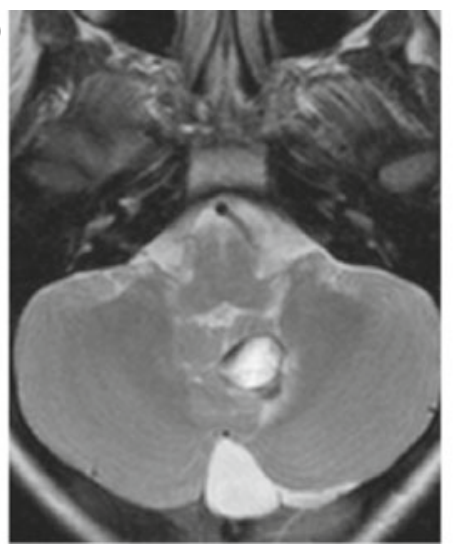

b)

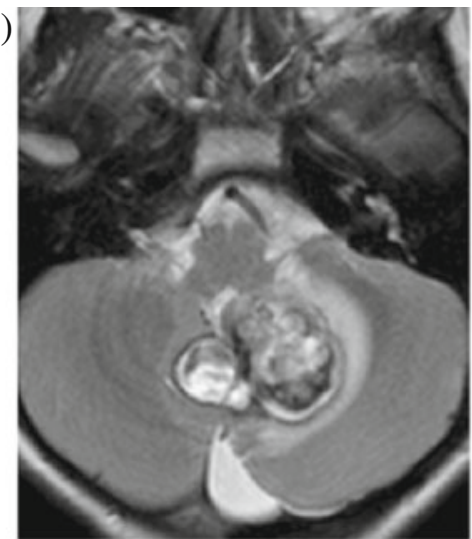

c)

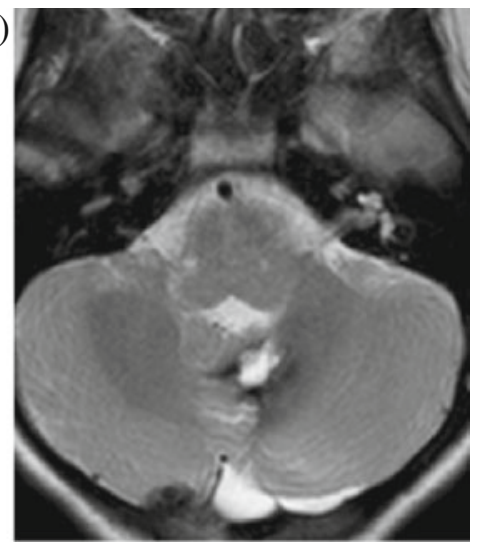

Fig. 2 MR images: Cavernoma of the cerebellum affecting mostly the vermis and the left cerebellar hemisphere in patient nos. 6 . The a baseline of T2-weighted MRI shows the characteristic of dark ring around the lesion (hemosiderin and/or calcification). The follow-up scan 5 months later $\mathbf{b}$ revealed significant progression in size and change in signal characteristics due to repetitive intra-lesional bleeding with progressive compression of the fourth ventricle. The cavernous angioma had to be operated upon with a good postoperative result, $\mathbf{c}$ the MRI 2 months after surgery showing regression of the mass effect on the fourth ventricle and some hemosiderin residues at the operative bed 
Table 2 Results of the neurological investigations carried out with all eight patients compared to norm

\begin{tabular}{|c|c|c|c|c|c|c|c|c|c|c|c|}
\hline \multirow[t]{2}{*}{ Functions } & \multirow[t]{2}{*}{ Test materials } & \multirow{2}{*}{$\begin{array}{l}\text { Mean (SD) } \\
\text { norm data }\end{array}$} & \multirow{2}{*}{$\begin{array}{l}\text { Mean (SD) } \\
\text { study simple }\end{array}$} & \multicolumn{8}{|c|}{ Patient nos. } \\
\hline & & & & 1 & 2 & 3 & 4 & 5 & 6 & 7 & 8 \\
\hline \multirow[t]{11}{*}{ Motor functions } & D-KEFS (motor speed) & $10(3)$ & 12 & 12 & 13 & 13 & 13 & 12 & 13 & 10 & 12 \\
\hline & Zurich Neuromotor Assessment (pure motor) & $0(1)$ & -0.4 & -1.7 & -2.2 & 1.7 & -0.3 & -0.4 & 0 & -0.8 & 0.9 \\
\hline & ZNA (adaptive fine motor) & $0(1)$ & -3.4 & -1 & -9.5 & 0.5 & -3 & -2.1 & -3.5 & -7 & -1.4 \\
\hline & ZNA (adaptive gross motor) & $0(1)$ & -2.7 & -1.4 & -4.6 & -0.9 & -2.1 & -1.7 & -4.1 & -4.9 & -1.5 \\
\hline & ZNA (static balance) & $0(1)$ & -1.4 & -0.3 & -2.1 & -1.6 & -2.7 & 0 & -1 & 0 & -3.1 \\
\hline & ZNA (associated movements) & $0(1)$ & 0.2 & 0.3 & 0.9 & 0.6 & 0.1 & 0.6 & 0.4 & -1 & -0.1 \\
\hline & ICARS (static score) & total 34 & 1 & 1 & 3 & 0 & 0 & 0 & 0 & 4 & 4 \\
\hline & ICARS (kinetic score) & total 52 & 5 & 5 & 5 & 3 & 1 & 0 & 10 & 4 & 5 \\
\hline & ICARS (dysarthria score) & total 8 & 0.4 & 0 & 1 & 0 & 0 & 0 & 0 & 1 & 1 \\
\hline & ICARS (oculomotor movements) & total 6 & 1.5 & 4 & 3 & 0 & 1 & 0 & 1 & 2 & 2 \\
\hline & ICARS (total ataxia) & total 100 & 7 & 10 & 12 & 3 & 2 & 0 & 11 & 11 & 8 \\
\hline
\end{tabular}

For the Zurich Neuromotor Assessment (ZNA), the scales have been converted into standard $z$-scores. Results that are more than one standard deviation below norm data (as referred in the Zurich Neuromotor Assessment test manual) are considered to be pathologic and are therefore printed in bold. Note that this table is just an overview of the collected results. Not all results are illustrated

\section{Questionnaires}

Results of the questionnaires on emotional well-being, possible behavior problems, and quality of life of the participants revealed no significant impairments or restrictions compared with healthy children or young adults. The questionnaires (CBCL and Kidscreen-52) found the participants to be in good general mental health with slightly reduced physical well-being and somatic complaints in some individuals. The majority, however, did not report that somatic symptoms influenced their daily activities. In general, the patients' self-ratings matched the parents' ratings quite well. In individual patients, the $\mathrm{CBCL} / \mathrm{YSR}$ scales for withdrawal, somatic complaints, social problems, and internalizing showed minor problems. However, there have been no reports of rule-breaking behavior or other externalizing problems. Patient nos. 2, 6, 7, and 8 (all with a lesioned vermis) were reported to have more behavioral problems and to be more affected by emotional disturbances than the other patients. In two participants (patient nos. 1 and 3 - both with an intact vermis), no psychiatric disturbances or behavioral problems were captured by the ratings. Quality of life, as assessed by the ILK-MARSYS, was rated to be average or even above average by all patients and their parents.

\section{Discussion}

The present study, with its selection of patients with histories of acquired non-neoplastic cerebellar lesions during childhood, provides a unique opportunity to address speculations about the developing cognitive cerebellum.
Not surprisingly, the neurologic examination revealed motor deficits in all patients, especially in fine and gross motor tasks (ZNA). Despite these results, the ICARS revealed only mild and quite heterogeneous signs of ataxia in our cohort. The ICARS has been shown to be a reliable and valid test for ataxia after cerebellar injuries, especially for assessing oculomotor functions [17, 42]. In the present patient group, the subscale for oculomotor movements has indeed been revealed to be the most sensitive ICARS subscale. These results confirm that the cerebellum is an important brain structure for (guided) eye movements. Such restricted voluntary eye movements might be the main cause of possible reading problems. Neuropsychologically based reading problems, due to problems in language processing, are probably secondary in patients with cerebellar injuries. However, in our patient group, no restrictions in reading accuracy and reading speed were observed.

The general intelligence assessments of the whole patient group revealed a mean FSIQ of 102 (SD 11). This result does not significantly differ from the general cognitive abilities of the healthy population. The analysis further revealed no significant correlation between time since injury (in years) and the FSIQ. However, we found a significant positive correlation between age at injury and FSIQ (Pearson correlation $0.779 ; p=0.023)$. This observation is in line with results obtained in studies with patients suffering from oncological problems, in which younger age at tumor resection is tied to a higher risk for compromised cognitive outcomes [24, 39]. Thus, our results support the idea in the literature that the child's cerebellum plays an important role for developing cognitive functions $[24,34,35,39]$. We assume that the cerebellum probably triggers the functional growth of other brain structures, e.g., the frontal lobe. With increasing 
Table 3 Results of the neuropsychological assessments carried out with all eight patients compared to norm

\begin{tabular}{|c|c|c|c|c|c|c|c|c|c|c|c|}
\hline \multirow[t]{2}{*}{ Cognitive function } & \multirow{2}{*}{$\begin{array}{l}\text { Cognitive/neuropsychological test } \\
\text { material }\end{array}$} & \multirow{2}{*}{$\begin{array}{l}\text { Norm data } \\
\text { mean (SD) } \\
\text { or PR }\end{array}$} & \multirow{2}{*}{$\begin{array}{l}\text { Study sample } \\
\text { mean (SD) } \\
\text { or PR }\end{array}$} & \multicolumn{8}{|c|}{ Patient nos. } \\
\hline & & & & 1 & 2 & 3 & 4 & 5 & 6 & 7 & 8 \\
\hline Intellectual ability & WISC-IV/WAIS-III full scale IQ & $100(15)$ & $102(11)$ & 110 & 98 & 108 & 83 & 101 & 108 & 91 & 118 \\
\hline \multirow{6}{*}{$\begin{array}{l}\text { Information } \\
\text { processing speed }\end{array}$} & WISC-IV/WAIS-III processing speed & $100(15)$ & $100(18)$ & 111 & 79 & 135 & 83 & 91 & 97 & 91 & 111 \\
\hline & WISC-IV/WAIS-III codes & $10(3)$ & $9(3)$ & 12 & 7 & 14 & 5 & 8 & 9 & 7 & 10 \\
\hline & WISC-IV/WAIS-III symbol search & $10(3)$ & $11(3)$ & 12 & 5 & 19 & 9 & 10 & 10 & 10 & 9 \\
\hline & TAP alertness phasic & $\mathrm{PR}=50$ & $\mathrm{PR}=35$ & $\mathrm{PR}=18$ & 8 & 12 & 69 & 50 & 38 & 46 & 42 \\
\hline & TAP alertness median with warning & $\mathrm{PR}$ & 27 & 10 & 16 & 16 & 24 & 58 & 31 & 16 & 46 \\
\hline & TAP alertness median without warning & PR & 31 & 12 & 34 & 42 & 21 & 46 & 42 & 16 & 46 \\
\hline \multirow[t]{9}{*}{ Attention processes } & TAP selective attention median & PR & 43 & 56 & 82 & 16 & 7 & 2 & 76 & 50 & 54 \\
\hline & TAP selective attention errors & PR & 39 & 43 & 12 & 59 & 54 & 54 & 24 & 24 & 43 \\
\hline & TAP selective attention omissions & PR & 39 & 50 & 50 & 50 & 50 & 50 & 50 & 8 & 4 \\
\hline & TAP divided attention median auditory & PR & 39 & 21 & 58 & 58 & 31 & 18 & 18 & 46 & 58 \\
\hline & TAP divided attention median visual & PR & 55 & 73 & 58 & 66 & 16 & 69 & 58 & 50 & 50 \\
\hline & TAP divided attention total errors & PR & 54 & 34 & 79 & 79 & 50 & 27 & 50 & 79 & 34 \\
\hline & TAP divided attention total omissions & PR & 55 & 90 & 84 & 27 & 34 & 38 & 21 & 95 & 50 \\
\hline & TAP incompatibility median & PR & 23 & 14 & 18 & 12 & 12 & 4 & 42 & 46 & 38 \\
\hline & TAP incompatibility errors & PR & 60 & 93 & 62 & 95 & 86 & 4 & 5 & 73 & 62 \\
\hline \multirow[t]{7}{*}{ Executive functions } & RWT semantic verbal fluency "animals" & PR & 33 & 23 & 93 & 60 & 2 & 32 & 10 & 5 & 35 \\
\hline & $\begin{array}{l}\text { RWT semantic verbal fluency "sports/ } \\
\text { fruits" }\end{array}$ & PR & 35 & 38 & 27 & 19 & 9 & 72 & 69 & 6 & 39 \\
\hline & RWT phonemic verbal fluency "s" & PR & 36 & 63 & 66 & 38 & 5 & 6 & 16 & 28 & 62 \\
\hline & Wisconsin Card Sorting Test (total errors) & PR & 69 & 92 & 77 & 92 & 7 & $\mathrm{n} / \mathrm{a}$ & 95 & 42 & 77 \\
\hline & WSCT (perseverative response) & PR & 69 & 99 & 87 & 99 & 7 & $\mathrm{n} / \mathrm{a}$ & 96 & 47 & 77 \\
\hline & WSCT (perseverative errors) & PR & 69 & 99 & 86 & 98 & 9 & $\mathrm{n} / \mathrm{a}$ & 96 & 50 & 73 \\
\hline & WSCT (non-perseverative errors) & PR & 60 & 73 & 63 & 84 & 8 & $\mathrm{n} / \mathrm{a}$ & 87 & 30 & 75 \\
\hline \multirow{8}{*}{$\begin{array}{l}\text { Verbal long-term } \\
\text { memory }\end{array}$} & RAVLT run 1 & PR & 56 & 25 & 68 & 80 & 90 & 63 & 18 & 4 & 96 \\
\hline & RAVLT run 5 & PR & 40 & 35 & 55 & 20 & 35 & 83 & 13 & 4 & 71 \\
\hline & RAVLT sum of $1-5$ & $50(10)$ & 56 & 48 & 63 & 56 & 60 & 54 & 37 & 33 & 96 \\
\hline & RAVLT interference trail & PR & 64 & 25 & 96 & 45 & 93 & 83 & 68 & 4 & 96 \\
\hline & RAVLT loss after interference & PR & 63 & 50 & 50 & 91 & 73 & 23 & 45 & 96 & 73 \\
\hline & RAVLT loss after $30 \mathrm{~min}$ & $50(10)$ & 63 & 63 & 66 & 66 & 66 & 50 & 60 & 66 & 63 \\
\hline & RAVLT recognition without mistakes & $50(10)$ & 53 & 54 & 54 & 53 & 54 & 68 & 52 & 36 & 54 \\
\hline & RAVLT perseveration & PR & 53 & 38 & 40 & 51 & 4 & 86 & 65 & 86 & 51 \\
\hline \multirow{3}{*}{$\begin{array}{l}\text { Visuo-spatial long- } \\
\text { term memory }\end{array}$} & REY figure reproduction & $50(10)$ & 46 & 66 & 62 & 55 & 36 & 44 & 27 & 23 & 56 \\
\hline & RVDLT recognition & PR & 75 & 100 & 100 & 85 & 80 & 65 & 75 & 15 & 80 \\
\hline & RVDLT correct & PR & 64 & 95 & 95 & 65 & 95 & 30 & 23 & 15 & 95 \\
\hline
\end{tabular}

Results that are more than one standard deviation below norm data (as referred in the test manuals) are considered to be pathologic and are therefore printed in bold. The scales used in the present table always match to scales in the particular test manual. This approach has been chosen as these scales are most familiar to clinicians who use the mentioned test. If three or more individuals scored at least one SD below the norm in a particular test, the test is considered to be sensitive. Note that only an aperture of mainly pathological results is illustrated. All results below PR16 (which refers to one SD below the mean of the healthy age-matched population) are considered to be pathologic

$P R$ percentile - mean $=50, n / a$ non-available-missing data

independence of these brain structures from cerebellar support, the importance of the cerebellum for cognitive functioning might decrease. This theory would explain why some adult patients have just minor or no cognitive problems after cerebellar injuries, but children with congenital cerebellar aplasia are most severely affected.
In this patient group, the biggest effect on neuropsychological functions can be seen in the domains of attention and information processing speed. Our results show that children and adolescents with cerebellar lesions are generally slower to react to stimuli (e.g., TAP alertness or TAP selective attention) than healthy children. If the patients perform at normal speed, 
they commit significantly more errors and omissions (Table 3 ). These results contrast with the findings of Haarmeier and Thier, who argue that significant results in most studies addressing the "attentive cerebellum" can be explained by oculomotor, motor, and/or working memory demands [43]. However, the slower reaction times on our tasks cannot be explained by oculomotor problems (as in these subtests no eye movements were required) or motor deficits (as the patients were instructed to have their hands already lying on the response button during the tests). In addition, the aforementioned tasks did not involve working memory.

Significantly slower reaction times in the majority of the patients (and in two cases, also significantly more errors) can further be seen in the TAP subtest for incompatibility when compared to their peers. This subtest is supposed to measure the subject's susceptibility to interference, and, in contrast to the tasks measuring reaction time, it consists of a working memory component. Susceptibility to interference is associated with compromised executive functions. Significant problems in the semantic and/or phonemic word fluency tests (RWT) further strengthen the observation of executive problems in these patients $[8,11-13]$. Taking these results and the diagnosed dysexecutive syndrome of patient nos. 4 into account supports speculations about alteration of the cerebello-frontal circuitry following cerebellar injuries [26, 39, 44].

Some studies imply that the crossing of cerebellar-cortical pathways leads to visuo-spatial problems after left cerebellar injuries and to language disturbances after injuries of the right cerebellar hemisphere, respectively [23, 24]. The present study did not reveal that any language problems had occurred after right cerebellar hemisphere lesions.

Even though support for the involvement of the cerebellum in affective and behavioral regulation processes has emerged from psychiatric studies and from studies on children with congenital cerebellar malformations, it is still unclear if these problems correspond to the phenotype of problems resulting from acquired cerebellar lesions during development [34-37, 45]. The results of the questionnaires revealed that the patients experienced just minor emotional distress. Any patients or parents who did report affective and behavioral regulation problems exclusively mentioned internalizing problems, rather than rule-breaking or aggressive behavior. In four of our eight patients, higher scores on emotional and behavioral problem scales coincided with an involvement of the vermis of the cerebellum in the injury. These observations are in line with reports that show a relationship between affective/behavior problems and an impairment of the vermis in the human cerebellum $[16,38]$.

\section{Limitations}

Even though the present study represents the largest ever reported cohort of pediatric patients with acquired hemor- rhagic cerebellar lesions, it still only consists of eight participants. Due to the small number of patients, we considered a healthy control group to be inappropriate. However, normative data of healthy children/adolescents were available from the test manuals. Any future study with more participants will need to consider whether a control group might be feasible. Furthermore, our patients sustained their injuries in a circumscribed time period. A topic of interest would be the effect on developing cognitive functions of a non-neoplastic lesion acquired early in life. In addition, cerebellar bleeding might only marginally cause necrosis. Thus, outcome after hemorrhagic cerebellar injury is probably much better compared with neoplastic space-occupying lesions that cause disruption of fibers. Moreover, in the present study, we looked at long-term outcome in adolescence or even early adulthood, and thus, potential short-term problems in these patients have been neglected.

\section{Conclusions}

All patients investigated in our study presented with mild and mostly subclinical motor problems that did not impact their activities on a daily basis. Cognitive impairments, if present, were mild in the majority but extremely heterogeneous in profile. This fact shows that detailed and individual-based neuropsychological evaluation is necessary in these patients. The circumscribed motor impairments and mild cognitive problems, which could only be evidenced in laboratory conditions, support the ideas that hemorrhagic lesions show marked acute symptoms, but have a promising long-term prognosis and good quality of life. Though, the presence of cerebellar cognitive affective syndrome, as described in the literature, was only expressed in children with vermis lesions. Children without vermis lesions have not been reported to show any behavioral or emotional disturbances. Our data show that young age at the time of injury and bi-hemispheric cerebellar lesions might be an important predictor for long-term cognitive outcome. Most likely, the developing cerebellum triggers the functional growth of other brain structures. With increasing independence of these brain structures from cerebellar support, the cerebellum becomes less important in processing neuropsychological functions and therefore, less vulnerable to injury.

Acknowledgments We thank all children/adolescents and parents involved in this project for their participation. We are also very thankful to Mrs. Angela Kummer and Mrs. Jacqueline Brunori for their helpful assistance in preparing the manuscript. There has been no conflict of interest. The study was funded by the Departments of Pediatric Neurology of the University Children's Hospitals of Bern and Zurich. 


\section{References}

1. Holmes G (1939) The cerebellum of man. Brain 62:1-30

2. Leiner HC, Leiner AL, Dow RS (1986) Does the cerebellum contribute to mental skills? Behav Neurosci 100:443-454

3. Bracke-Tolkmitt R, Linden A, Canavan AGM, Rockstroh B, Scholz E, Wessel K et al (1989) The cerebellum contributes to mental skills. Behav Neurosci 103(2):442-446

4. Mariën P, Baillieux H, De Smet HJ et al (2009) Cognitive, linguistic and affective disturbances following a right superior cerebellar artery infarction: a case study. Cortex 45:527-536

5. Ackermann H, Mathiak K, Riecker A (2007) The contribution of the cerebellum to speech production and speech perception: clinical and functional imaging data. Cerebellum 6:202-213

6. Ben-Yehudah G, Fiez JA (2008) Impact of cerebellar lesions on reading and phonological processing. Ann NY Acad Sci 1145:260-1174

7. Murdoch BE (2009) The cerebellum and language: historical perspective and review. Cortex 46(7):858-868

8. Kalashnikova LA, Zueva YV, Pugacheva OV, Korsakova NK (2005) Cognitive impairments in cerebellar infarcts. Neurosci Behav Physiol 35(8):773-779

9. Molinari M, Leggio MG (2007) Cerebellar information processing and visuospatial functions. Cerebellum 6:214-220

10. Hoffmann M, Schmitt F (2004) Cognitive impairment in isolated subtentorial stroke. Acta Neurol Scand 109:14-24

11. Ravizza SM, McCormick CA, Schlerf JE, Justus T, Ivry RB, Fiez JA (2006) Cerebellar damage produces selective deficits in verbal working memory. Brain 129:306-320

12. Whigham KB, O'Toole K (2007) Understanding the neuropsychologic outcome of pediatric AVM within a neurodevelopmental framework. Cognit Behav Neurol 20:244-257

13. Ben-Yehudah G, Guediche S, Fiez JA (2007) Cerebellar contributions to verbal working memory: beyond cognitive theory. Cerebellum 6:193-201

14. Leggio MG, Tedesco AM, Chiricozzi FR, Clausi S, Orsini A, Molinari M (2008) Cognitive sequencing impairment in patients with focal or atrophic cerebellar damage. Brain 131:1332-1343

15. Molinari M, Chiricozzi FR, Clausi A, Tedesco AM, De Lisa M, Leggio MG (2008) Cerebellum and detection of sequences, from perception to cognition. Cerebellum 8:611-615

16. Schmahmann JD, Sherman JC (1998) The cerebellar cognitive affective syndrome. Brain 121:561-579

17. Timmann D, Konczak J, Ilg W, Domchin O, Hermsdörfer J, Gizewski ER, Schoch B (2009) Current advances in lesionsymptom mapping of the human cerebellum. Neuroscience 162 (3):836-851

18. Kim SG, Ugurbil K, Strick P (1994) Activation of a cerebellar output nucleus during cognitive processing. Science 265:949951

19. Hu D, Shen H, Zhou Z (2008) Functional asymmetry in the cerebellum. A brief review. Cerebellum 7(3):304-313

20. Schmahmann JD, Pandya DN (1997) The cerebrocerebellar system. Int Rev Neurobiol 41:31-60

21. Middleton FA, Strick PL (1994) Anatomical evidence for cerebellar and basal ganglia involvement in higher cognitive function. Science 266:485-461

22. Thach WT (2007) On the mechanism of cerebellar contributions to cognition. Cerebellum 6:163-167

23. Gasparini M, DiPiero C, Ciccarelli O, Cacioppo MM, Pantano P, Lenzi GL (1999) Linguistic impairment after right cerebellar stroke: a case report. Eur J Neurol 6:353-356

24. Scott RB, Stoodley CJ, Anslow P, Stein J, Sugden EM, Mitchell CD (2001) Lateralized cognitive deficits in children following cerebellar lesions. Dev Med Child Neurol 43:685-691
25. Riva D (2000) Cerebellar contribution to behaviour and cognition in children. J Neurolinguist 13:215-225

26. Schmahmann JD, Caplan D (2006) Cognition, emotion and the cerebellum. Brain 129:288-292

27. Gordon N (2007) The cerebellum and cognition. Eur J Paediatr Neurol 11:232-234

28. Baillieux H, De Smet HJ, Lesage G, Paquier P, Deyn D, Mariën P (2006) Neurobehavioral alternations in an adolescent following posterior fossa tumor resection. Cerebellum 5:289-295

29. Levinsohn L, Cronin-Golomb A, Schmahmann JD (2000) Neuropsychological consequences of cerebellar tumor resection in children: cerebellar cognitive affective syndrome in a paediatric population. Brain 123:1041-1050

30. Charalambides C, Dinopoulos A, Sgouros S (2009) Neuropsychological sequelae and quality of life following treatment of posterior fossa ependymomas in children. Childs Nerv Syst 25 (10):1313-1320

31. Kossorotoff M, Gonin-Flambois C, Gitiaux C, Quijano S, Boddaert N, Bahi-Buisson N et al (2010) A cognitive and affective pattern in posterior fossa strokes in children: a case series. Dev Med Child Neurol 52(7):626-831

32. Riva D, Giorgi C (2000) The cerebellum contributes to higher functions during development: evidence from a series of children surgically treated for posterior fossa tumours. Brain 123:10511061

33. Shevell MI, Majnemer A (1996) Clinical features of developmental disability associated with cerebellar hypoplasia. Pediatr Neurol 15:224-229

34. Steinlin M (2007) The cerebellum in cognitive processes: supporting studies in children. Cerebellum 6:237-241

35. Steinlin M, Schmid M, Landau K, Boltshauser E (1997) Follow-up in children with Joubert syndrome. Neuropediatrics 28(4):204-211

36. Volpe JJ (2009) Cerebellum of the premature infant: rapidly developing, vulnerable, clinically important. J Child Neurol 24 (9):1085-1104

37. Messerschmidt A, Fuiko R, Prayer D, Brugger PC, Boltshauser E, Zoder G, Sterniste W, Weber M, Birnbacher R (2008) Disrupted cerebellar development in preterm infants is associated with impaired neurodevelopmental outcome. Eur J Pediatr 167 (10):1141-1147

38. Tavano A, Grasso R, Gagliardi C, Triulzi F, Bresolin N, Fabbro F, Borgatti R (2007) Disorders of cognitive and affective development in cerebellar malformations. Brain 130:2646-2660

39. Steinlin M, Imfeld S, Zulauf P, Boltshauser E, Lövblad KO, Ridolfi Lüthy A, Perrig W, Kaufmann F (2003) Neuropsychological long-term sequelae after posterior fossa tumour resection during childhood. Brain 126:1988-2008

40. Steinlin M, Pfister I, Pavlovic J, Everts R, Boltshauser E, Capone Mori A et al (2005) The first three years of the Swiss Neuropaediatric Stroke Registry (SNPSR): a population-based study of incidence, symptoms and risk factors. Neuropediatrics 36 (2): $90-97$

41. Trouillas P, Takayanagi T, Hallett M, Currier RD, Subramony SH, Wessel $\mathrm{K}$ et al (1997) International Cooperative Ataxia Rating scale for pharmacological assessment of the cerebellar syndrome. The Ataxia Neuropharmacology Committee of the World Federation of Neurology. J Neurol Sci 145:205-211

42. Schoch B, Regel JR, Frings M et al (2007) Reliability and validity of ICARS in focal cerebellar lesions. Mov Disord 22(15):2162-2169

43. Haarmeier T, Thier P (2007) The attentive cerebellum - myth or reality? Cerebellum 6:177-183

44. Bellebaum C, Daum I (2007) Cerebellar involvement in executive control. Cerebellum 6:184-192

45. Rapoport M, van Reekum R, Mayberg H (2000) The role of the cerebellum in cognition and behavior: a selective review. $\mathrm{J}$ Neuropsychiatr Clin Neurosci 12:193-198 


\section{References to test materials and questionnaires}

5-point Test: Regard M, Stauss E, Knapp P (1982) Children`s production of verbal and nonverbal fluency tasks. Perceptual and Motor Skills 55: 839-844

Child Behaviour Check List (CBCL) and Youth Self Report (YSR): Achenbach TM (1991) Manual for the Child Behavior Checklist/ 4-18 and the 1991 Profile. Burlington, USA: University of Vermont, Department of Psychiatry

Corsi Block Tapping Test (CORSI): Milner B (1971) Interhemispheric differences in the localization of psychological processes in man. British Medical Bulletin 27, 273-277

Delis-Kaplan Executive Function System (D-KEFS): Delis DC, Kaplan E, Framer IH (2001) Delis-Kaplan Executive Function System. San Antonio, USA: Psychological Corporation. Harcourt Brace and Company

International Cooperative Ataxia Rating Scale (ICARS): Trouillas P, Takayanagi T, Hallett M, et al. (1997) International Cooperative Ataxia Rating scale for pharmacological assessment of the cerebellar syndrome. The Ataxia Neuropharmacology Committee of the World Federation of Neurology. Journal of Neurological Sciences 145: 205-211

Kidscreen-52 questionnaire: Ravens-Sieberer U, Gosch A, Abel T, Auquier P, Bellach BM, Bruil J, and the European Kidscreen Group (2001) Quality of life in children and adolescents: a European public health perspective. Social and Preventive Medicine 46: 297-302

Marburger inventory to establish the quality of life in children and adolescents (ILK-MARSYS): Mattejat F, Remschmidt H (2006) Inventar zur Erfassung der Lebensqualität bei Kindern und Jugendlichen. Ratingbogen für Kinder, Jugendliche und Eltern. Bern, Switzerland: Verlag Hans Huber. (in German)
Regensburger Word Fluency Test (RWT): Aschenbrenner S, Tucha O, Lange KW (2000) Regensburger Word Fluency Test (German version). Göttingen, Germany: Hogrefe. (in German)

Rey Auditory Verbal Learning Test (RAVLT): Rey A (1964) L'examen clinique en psychologie. Paris: Presses Universitaire de France. (in French)

Rey Osterrieth Complex Figure (REY figure): Rey A (1941) L'examen psychologique dans les cas dencephalopathie traumatique. Archives de Psychologie 28,286-340. (in French)

Waber D, Holmes JM (1985) Assessing children's copy productions of the Rey Osterrieth Complex Figure. Journal of Clinical and Experimental Neuropsychology 7: 264-280

Rey Visual Design Learning Test (RVDLT): Rey A (1964) L'examen clinique en psychologie. Paris: Presses Universitaire de France. (in French)

Test battery of attentional performance (TAP): Zimmermann P, Fimm B (2002) TAP Testbatterie zur Aufmerksamkeitsprüfung. Herzogenrath, Germany: Psytest. (in German)

Wechsler Intelligence Scale for Adults, 3rd edition (WAIS-III): Wechsler D (1999) Wechsler Abbreviated Scales of Intelligence (German version). San Antonio, USA: Psychological Corporation. Harcourt Brace and Company

Wechsler Intelligence Scale for Children, 4th edition (WISC-IV): Wechsler D (1991) Wechsler Intelligence Scales for children (German version). San Antonio, USA: Psychological Corporation. Harcourt Brace and Company

Wisconsin Card Sorting Test (WSCT): Psychological Assessment Resources (2003) Computerized Wisconsin Card Sort Task Version 4 (WCST). Lutz, USA: Psychological Assessment Resources

Zurich Neuromotor Assessment battery (ZNA): Largo RH, Fischer J, Caflisch J (2002) Zurich Neuromotor Assessement. Zurich, Switzerland: AWE Verlag. (in German) 